\title{
RAPID SACRIFICIAL GERMANIUM ETCHING USING XENON DIFLUORIDE
}

\author{
Garrett D. Cole and Elaine Behymer
}

Center for Micro- and Nanotechnologies, Lawrence Livermore National Laboratory, 7000 East Avenue, Livermore, California 94550, USA

\begin{abstract}
We present a novel micromachining procedure employing the noble gas halide, xenon difluoride $\left(\mathrm{XeF}_{2}\right)$, to rapidly undercut a sacrificial layer comprised of low-temperature deposited amorphous germanium $(\alpha-\mathrm{Ge})$. As a proof of concept, this process is utilized to fabricate electrostatically-actuated suspended Bragg mirrors applicable to wavelength-tunable surface-normal photonic devices. Exploiting $\mathrm{XeF}_{2}$ as the release etchant, we observe a truly remarkable lateral etch rate in excess of $150 \mu \mathrm{m} / \mathrm{min}$ for a $1-\mu \mathrm{m}$ thick sacrificial $\alpha$-Ge film. Given the rapid nature of this etch, we envision this procedure to be a promising approach for the lateral undercutting of large-area microdevices.
\end{abstract}

\section{INTRODUCTION}

The sacrificial etch method is a fundamental technique for the construction of microelectromechanical systems (MEMS). Within this framework, processes employing gas-phase etchants such as $\mathrm{XeF}_{2}$, bromine trifluoride [1], and $\mathrm{HF}$ vapor [2] have proven quite successful; particularly in the case of $\mathrm{XeF}_{2}$ for sacrificial silicon etching. As opposed to wet chemistry mediated undercutting, these processes enable rapid lateral etching without inducing capillary forces, thus eliminating the need for post-release critical point drying (CPD) and enabling efficient penetration into narrow micro/nanoscale vias. In this manuscript we present a novel micromachining procedure utilizing $\mathrm{XeF}_{2}$ to selectively etch a sacrificial $\alpha$-Ge film. When compared with sacrificial silicon etching, this process exhibits a significant enhancement in the achievable lateral etch rate. Additionally, the fabrication sequence may be tailored to incorporate low temperature deposition steps, ranging from thermal or electron-beam evaporation of $\alpha$-Ge at room temperature (enabling deposition rates of $100 \AA / \mathrm{s}$ [3]), to CVD processes producing high quality polycrystalline germanium films at temperatures below $450^{\circ} \mathrm{C}$ [4].

\section{GAS-PHASE GERMANIUM ETCHING}

The results from a preliminary investigation of the lateral etch rate of $\alpha-\mathrm{Ge}$ in $\mathrm{XeF}_{2}$ are presented in Fig. 1. For this experiment the $\alpha-G e$ is deposited by room temperature electron-beam evaporation onto single-crystal GaAs substrates. This procedure results in extremely smooth films with surface roughness values ranging from 8-11 Å. Following evaporation, a $\mathrm{SiN}_{\mathrm{x}}$ structural film is deposited via PECVD at $200^{\circ} \mathrm{C}$. A pattern consisting of circular etch vias and orthogonal stripes is then transferred into the $\operatorname{SiN}_{x}$ with dilute HF. Finally, the $\alpha-G e$ layer is selectively undercut in a pulsed $\mathrm{XeF}_{2}$ etching system [5]. From the circular vias we measure a lateral etch rate of approximately $55 \mu \mathrm{m} / \mathrm{min}$ for the 1 - $\mu$ m-thick $\alpha$-Ge sample. This value is comparable to that recently presented for crystalline germanium samples [6] and largely exceeds the maximum typical silicon etch rate of approximately $10 \mu \mathrm{m} / \mathrm{min}$ [7]. However, for the $30-\mu \mathrm{m}$-wide orthogonal stripes surrounding the circular apertures (see inset image in Fig. 1), we measure a remarkable lateral etch rate of nearly $160 \mu \mathrm{m} / \mathrm{min}$. Given the rapid nature of this process, we propose that $\mathrm{XeF}_{2}$ etching of germanium is a promising approach for undercutting large-area devices or for the lift-off of continuous films from a potentially re-usable handle substrate, as in the epitaxial liftoff process pioneered by Yablonovitch [8].

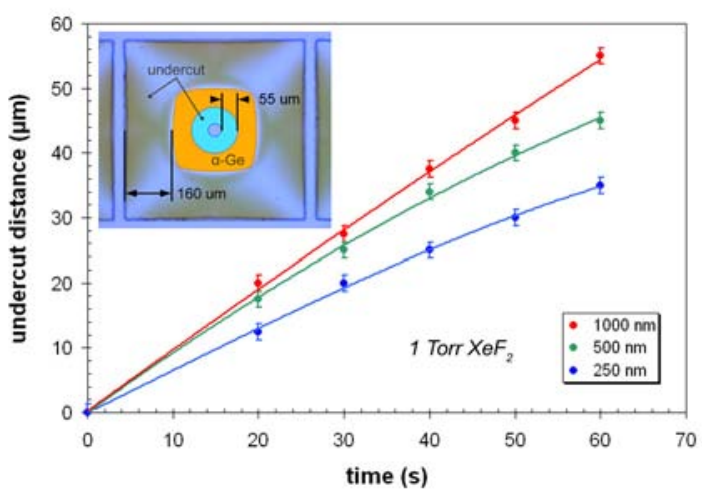

Figure 1: Undercut distance as a function of time for various thickness $\alpha$-Ge films. The data corresponds to the position of the lateral etch front with respect to the 40- $\mu$ m-diameter etch vias. Inset: micrograph of 1- $\mu \mathrm{m} \alpha$-Ge sample after 60 s of etching.

\section{SUSPENDED DIELECTRIC BRAGG MIRRORS}

The viability of our novel micromachining process is demonstrated through the development of electrostatically-actuated suspended dielectric Bragg reflectors (see Fig. 2), applicable to wavelength-tunable vertical-cavity lasers (VCSELs) $[9,10]$ and amplifiers [11]. The detailed fabrication sequence for these devices may be found in [12]. Although $\mathrm{XeF}_{2}$ has been applied extensively in silicon-based microfabrication, to the best of our knowledge, this is the first use of this etchant for the development of MEMS with a germanium sacrificial layer, as well as the first use of $\mathrm{XeF}_{2}$ for MEMS on a compound semiconductor substrate.

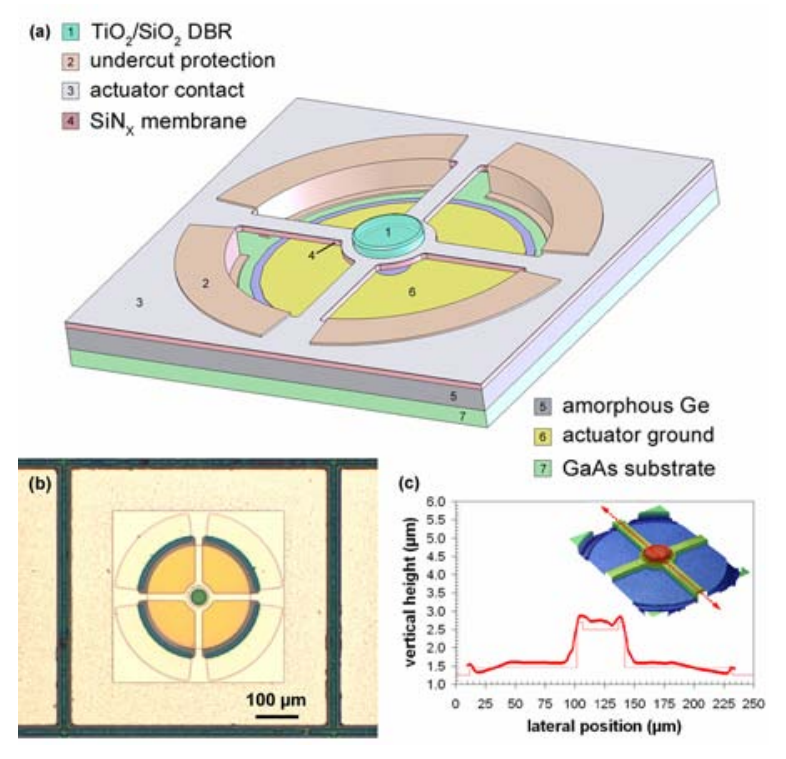

Figure 2: (a) Solid model of the suspended Bragg mirror. (b) Optical micrograph of a completed micromirror. (c) Line scan recorded with an optical profilometer; the inset includes the original 3-D mapping and indicates the path of the 2-D plot. The ideal profile is depicted along with the measurement. A minor upward bowing extends the nominal 1000-nm air gap to $1150 \mathrm{~nm}$. 
As with sacrificial silicon etching, this process results in excellent selectivity over most metals, dielectrics, polymers, etc. Additionally, the use of the non-plasma etch eliminates surface damage induced by ion bombardment, while the simplicity of the etching system allows for in situ viewing of the lateral etch front. Compared with previous implementations [11], the fabrication of the suspended mirror structures is greatly simplified through: 1) the elimination of the CPD process, 2) the ability to monitor the lateral etch front beneath the transparent mirror structure, and 3) the largely reduced release etch time. In fact, for the devices presented in this manuscript, the total time required to successfully undercut an 80- $\mu \mathrm{m}$ diameter membrane is less than 20 seconds (achieved through two 10-s pulses or one single 20-s etch pulse).

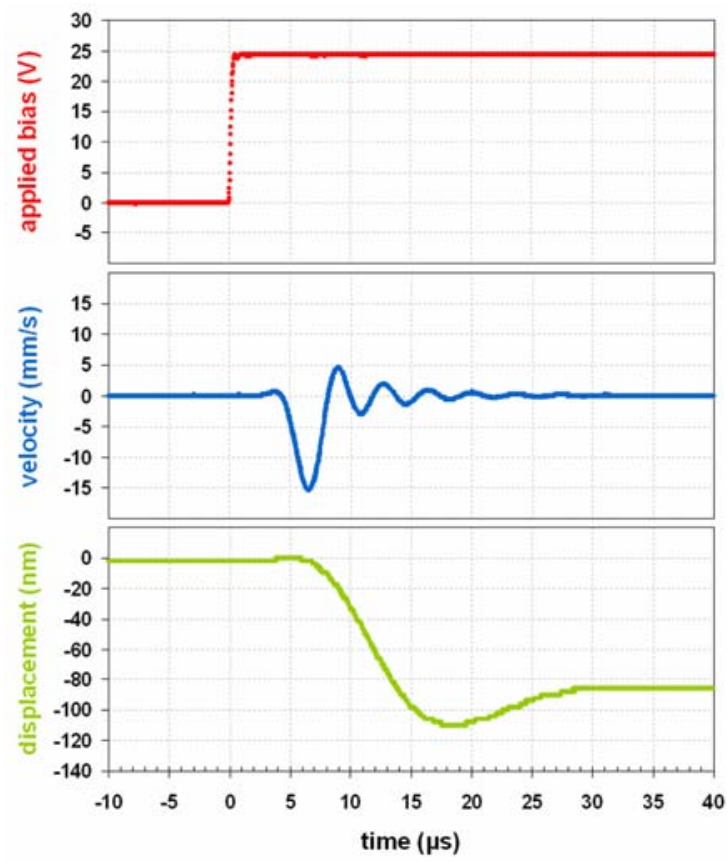

Figure 3: Step response (atmospheric pressure) for a $60 \mu \mathrm{m}$ diameter membrane with $70 \mu \mathrm{m}$ long by $20 \mu \mathrm{m}$ wide tethers at a bias of $24 \mathrm{~V}$; negative values indicate vertical motion towards the substrate. Ringing leads to a settling time of just under $30 \mu \mathrm{s}$.

Following fabrication we investigate the static and dynamic characteristics of the suspended reflector using a laser-Dopplervibrometer-based MEMS motion characterization system [13]. Fig. 3 presents the step response of the suspended mirror structure, while the compiled displacement versus bias data are included in Fig. 4. It is important to note that the step response exhibits temporal smoothing due to the limited 3-dB bandwidth of the vibrometer (250 kHz for velocity and $50 \mathrm{kHz}$ for displacement). The settling time of this structure may be reduced by optimizing the geometry of the actuator to achieve sufficient squeeze-film damping for a near critically-damped response [14].

From the theoretical fit of the static deflection data, the estimated snap-down voltage (displacement) is $30.9 \mathrm{~V}(447 \mathrm{~nm})$ with the best fit achieved for an air-gap thickness of $1120 \mathrm{~nm}$. This value compares well with the profilometer measurements of the air-gap thickness $(1150 \mathrm{~nm})$. Assuming this mirror is incorporated on a GaAs-based 1- $\lambda$ semiconductor cavity with a hypothetical center wavelength of $1000 \mathrm{~nm}$, the measured deflection would result in a wavelength tuning range of $37 \mathrm{~nm}$ at $30 \mathrm{~V}$ bias (this calculation includes the penetration depth for a 25.5 period GaAs/AlAs bottom mirror).

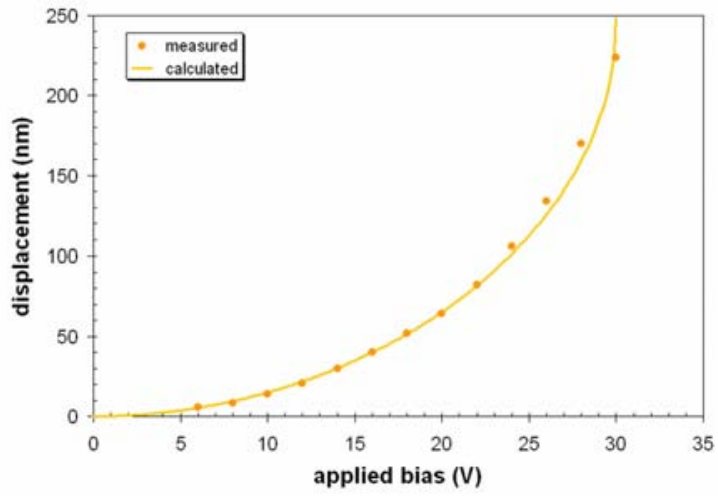

Figure 4: Static deflection characteristics of the suspended mirror. The theoretical curve is generated from the iterative solution of a one-dimensional electromechanical model [12]. Current-voltage measurements demonstrate leakage currents below $20 \mathrm{pA}$ at $30 \mathrm{~V}$.

\section{SUMMARY AND FUTURE WORK}

We have demonstrated a novel micromachining process exploiting $\mathrm{XeF}_{2}$ to rapidly remove an evaporated $\alpha$-Ge sacrificial layer. For large etch apertures we record undercut rates exceeding $150 \mu \mathrm{m} / \mathrm{min}$. Further investigation will be necessary to quantify the lateral etch rate for narrow (sub-micron) vias. Finally, this procedure has been utilized to demonstrate high-performance arrays of electrostatically actuated suspended Bragg reflectors.

\section{ACKNOWLEDGMENTS}

This work performed under the auspices of the U.S. DOE by Lawrence Livermore National Laboratory under Contract DEAC52-07NA27344. GDC thanks Professor Roger T. Howe of Stanford University for a number of insightful discussions.

\section{REFERENCES}

[1] T. Yao, X. Yang, Y.C. Tai, Sens. Actuators A 97, 652 (2002).

[2] Y.I. Lee, K.H. Park, J. Lee, C.S. Lee, H.J. Yoo, C.J. Kim, Y.S. Yoon, J. MEMS 6, 226 (1997).

[3] M.L. Rappaport, B. Berkovitz, J. Vac. Sci. Technol. 21, 102 (1982).

[4] A.E. Franke, J.M. Heck, T.J. King, R.T. Howe, J. MEMS 12, 160 (2003).

[5] P.B. Chu, J.T. Chen, R. Yeh, G. Lin, J.C.P. Huang, B.A. Warneke, S.J. Pister, Proc. Transducers '97, Chicago, IL, USA, paper 2D3.01, p. 665.

[6] G. Xuan, T.N. Adam, J. Suehle, E. Fitzgerald, P. Lv, N. Sustersic, M.J. Coppinger, J. Kolodzey, Proc. ISTDM 2006, Princeton, NJ, paper 5P.44.

[7] K.R. Williams, R.S. Muller, J. MEMS 5, 256 (1996).

[8] E. Yablonovitch, D.M. Hwang, T.J. Gmitter, L.T. Florez, J.P. Harbison, Appl. Phys. Lett. 56, 2419 (1990).

[9] M. Maute, B. Kögel, G. Bohm, P. Meissner, M.-C. Amann, IEEE Photon. Technol. Lett. 18, 688 (2006).

[10] M.C. Huang, K.B. Cheng, Y. Zhou, A.P. Pisano, C.J. ChangHasnain, IEEE J. Sel. Top. Quantum Electron. 13, 374 (2007).

[11] G.D. Cole, E.S. Björlin, C.S. Wang, N.C. MacDonald, J.E. Bowers, IEEE Photon. Technol. Lett. 17, 2526 (2005).

[12] G.D. Cole, E. Behymer, L.L. Goddard, T.C. Bond, J. Vac. Sci. Technol. B 26, 593 (2008).

[13] K.L. Turner, P.G. Hartwell, N.C. Macdonald, Proc. Transducers '99, Sendai, Japan, p. 1144.

[14] G.D. Cole, J.E. Bowers, K.L. Turner, N. C. MacDonald, IEEE/LEOS Optical MEMS 2005, Oulu, Finland, paper F4. 